\title{
P53-dependent radiosensitizing effects of Hsp90 inhibitor 17-Allylamino-17-demethoxygeldanamycin on human oral squamous cell carcinoma cell lines
}

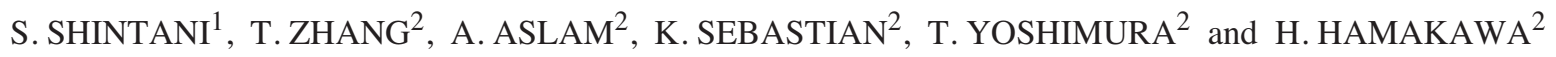 \\ ${ }^{1}$ Department of Oral and Maxillofacial Surgery, Showa University School of Dentistry, Ohta-Ku 145-8515, Tokyo; \\ ${ }^{2}$ Department of Oral and Maxillofacial Surgery, Ehime University School of Medicine, Ehime, Japan
}

Received April 27, 2006; Accepted June 29, 2006

\begin{abstract}
Development of new molecular target therapeutic agents is expected to improve clinical outcome, ideally with efficacy in both single and combined treatment modalities. Because of the potential for affecting multiple signaling pathways, inhibition of the molecular chaperone heat shock protein 90 (Hsp90) may provide a strategy for enhancing tumor cell radiation sensitivity. Therefore, we have investigated the effects of Hsp90 inhibitor 17-Allylamino-17-demethoxygeldanamycin (17-AAG) on radiation sensitivity of human tumor cells in vitro. We evaluated the effects of 17-AAG using oral squamous cell carcinoma (OSCC) cell lines (HSC2, HSC3 and HSC4), including two types of SAS cells with a wild-type (SAS/neo), or a mutated p53 status (SAS/Trp248). Apoptosis and clonogenic survival were examined after exposure of the cells to radiation. For mechanistic insight, we analyzed cell cycle, several signaling factors and molecular markers including Akt, Raf-1, p38 MAPK, Cdc25B, Cdc25C, Cdk2 and p21. Treatment of OSCC cell lines with 17-AAG resulted in cytotoxicity and, when combined with radiation, enhanced the radiation response. However, the responses depended on p53 status. 17-AAG enhanced the radiation sensitivity significantly and induced apoptosis in the SAS/neo cell which has a wild-type p53. But the radiation sensitizing effect of 17-AAG was limited in the SAS/Trp248 cell which has a mutated p53. We also measured the total levels of several prosurvival and cell cycle signaling proteins. Akt, Raf-1 and Cdc25C expression were down-regulated in 17-AAG-treated cells. These data indicate that 17-AAG inhibits the proliferation and enhances the radiation sensitivity of human OSCC cells in various levels. However, enhancement of radiation sensitivity by the Hsp90 inhibitor depended on p53 status. Therefore,
\end{abstract}

Correspondence to: Dr Satoru Shintani, Department of Oral and Maxillofacial Surgery, Showa University School of Dentistry, 1-1 Kitasenzoku 2-choume, Ohta-Ku, Tokyo 145-8515, Japan

E-mail: shintani@senzoku.showa-u.ac.jp

Key words: heat shock protein 90, oral cancer, p53 status, radiation
Hsp90 therapy combined with radiation might synergize with conventional therapies in patients with wild-type p53.

\section{Introduction}

The clinical outcome of oral cancer is expected to improve with the development of new molecular target therapeutic agents, on their own and in combination with existing treatment modalities. We specifically focused on the molecular chaperone heat shock protein 90 (Hsp90). Hsp90 is a unique chaperone and has been implicated in maintaining the conformation, stability and function of key client proteins involved in signal transduction pathways related to proliferation, cell cycle progression, and apoptosis. Hsp90 is also involved in malignant phenotype characteristics, including invasion, angiogenesis and metastasis $(1,2)$. In addition to signaling proteins such as steroid hormone receptors, Src family kinases and certain cyclin-dependent kinases, Hsp90 client proteins also include proteins associated with the radiation response, such as Akt and Raf-1 $(3,4)$. Hsp90 expression is up-regulated in tumors such as oral cancer, implying tumor cells may be preferentially affected by Hsp90-targeted therapies $(5,6)$. Therefore, Hsp90 represents a potentially attractive target for specific molecular anticancer agents.

17-AAG has significant anticancer properties; in fact, current clinical trials are investigating it as a sole treatment $(7,8)$. This agent disrupts the association of Hsp90 with client proteins by occupying the nucleotide-binding site of Hsp90, thereby preventing binding of Hsp90 with ATP and profoundly affecting the composition of Hsp90-containing multimolecular chaperone complexes (9-11). Interestingly, tumor cells increase Hsp90 levels, and many cytoprotective and prosurvival pathways are functionally stabilized by $\operatorname{Hsp} 90(5,6,12)$. 17-AAG induces proteasomal degradation of a subset of Hsp90 client proteins involved in signal transduction and oncogenes known to be involved in transformation; however, these compounds have little effect on the untransformed counterparts of malignant tissues (13-16). Collectively, these findings suggest that targeting Hsp90 will preferentially destroy abnormal cells and spare normal tissue.

While the molecular cell survival mechanism after radiation exposure has not been completely elucidated, it is clear that the DNA repair, cell cycle checkpoint activation and 
apoptosis pathways are related to radiation response. Multiple independent and interacting processes can regulate radiationinduced cell death since control over a variety of signaling pathways is related to radiation response. Current efforts to develop strategies for enhancing tumor radiation sensitivity have focused on agents that target a single molecule. However, assuming that a combined process determines radiation sensitivity, a more effective approach would be to target multiple radiation response regulatory molecules. Recent reports showed that the Hsp90 inhibitor enhances the radiation sensitivity of human tumor cell lines (17-19). Therefore, we hypothesized that Hsp90 inhibition by 17-AAG might increase radiation-induced tumor cell death.

The effect a specific molecule has on radiation response often depends on the genetic background of the tumor cell, for example p53 $(20,21)$. Tumor cells with an altered p53 are more sensitive than cells expressing wild-type p53 $(22,23)$. Thus, using two OSCC cell lines, one with a wild-type and the other with mutated p53 status, we evaluated how 17-AAG effects the radiation response. In addition, we analyzed several prosurvival signaling factors or molecular markers, including Akt1, Raf-1 and p38 MAPK expression.

\section{Materials and methods}

Immunohistochemical analysis. Hsp90 expression was assessed in histological sections of OSCC tissues. Twenty tumor specimens were fixed in $10 \%$ neutral-buffered formalin. After embedding in paraffin, $5-\mu \mathrm{m}$ sections were cut and mounted. Sections were then dried, de-paraffinized and rehydrated. After quenching endogenous peroxidase activity and blocking non-specific binding sites, slides were incubated at $4{ }^{\circ} \mathrm{C}$ overnight with 1:100 dilution of primary antibody directed against Hsp27, Hsp70, and Hsp90 (Santa Cruz Biotech, Santa Cruz, CA). Immunostaining was performed using the Envision system (Dako, Japan) according to the manufacturer's instructions. Peroxidase activity was visualized by applying diaminobenzidine chromogen containing $0.05 \%$ hydrogen peroxidase. The sections were then counterstained with hematoxylin, dehydrated, cleared and finally mounted.

Cell culture, 17-AAG treatment, and radiation. OSCC cells (HSC2, HSC3, HSC4, SAS/neo, SAS/Trp248) and HaCaT were grown in Ham/F12: Dulbecco's modified Eagle's medium (1:1) (Gibco-BRL, Gaithersburg, MD, USA) supplemented with $10 \%$ heat-inactivated $\left(56^{\circ} \mathrm{C}, 30 \mathrm{~min}\right)$ calf serum. HSC2, HSC 3 and HSC4 cells were obtained from Japanese Collection Research Bioresourses, and SAS/neo and SAS/Trp248 cells were obtained from Dr T. Ohnishi (Nara Medical University) (24). Medium was supplemented with penicillin (100 units/ml) and streptomycin $(100 \mu \mathrm{g} / \mathrm{ml})$. All cells were plated at densities of $2 \times 10^{5}$ cells $/ 100-\mathrm{mm}$ dish, grown in a humidified $5 \% \mathrm{CO}_{2}$ incubator at $37^{\circ} \mathrm{C}$, and passaged at $75 \%$ confluence. $17-\mathrm{AAG}$ (A-1256: A.FG. Scientific Inc., San Diego, CA, USA) was dissolved in DMSO to yield 10-mM stock solutions and then stored at $-80^{\circ} \mathrm{C}$. Agents were added to the growth medium of designated cells to achieve specific molar concentrations, after which they were returned to $37^{\circ} \mathrm{C}$. Corresponding volumes of DMSO vehicle were added to designated sham controls in each experiment.
Western blot analysis. Primary antibodies against Hsp27, Hsp70, Hsp90 and actin were obtained from Santa Cruz Biotech, while antibodies against Akt 1, Raf 1 and p38/MAPK were obtained from Cell Signaling Technology (Beverly, MA). Control and 17-AAG (100 nM)-treated cells were irradiated with 4 Gy and cells were lysed after a 12-h incubation period. To analyze the effects of $17-\mathrm{AAG}(100 \mathrm{nM})$ and/or radiation (4 Gy) on signal transduction pathways and cell cycle related proteins, OSCC cells were lysed in buffer (1X PBS, $1 \%$ Nonidet-P 40, $0.5 \%$ sodium deoxycholate, deoxycholate and $0.1 \% \mathrm{SDS}$ ) containing protease, $0.1 \mathrm{M} \mathrm{NaF}, 10 \mathrm{mg} / \mathrm{ml}$ leupeptin (Sigma, Tokyo, Japan), $0.1 \mathrm{mg} / \mathrm{ml}$ trypsin inhibitor (Sigma, Tokyo, Japan), $0.1 \mathrm{mg} / \mathrm{ml}$ aprotinin (Sigma, Tokyo, Japan) and $50 \mathrm{mg} / \mathrm{ml}$ PMSF (Sigma). Following sonication and incubation on ice for $30 \mathrm{~min}$, the supernatant was collected. Protein concentrations were determined using a DC protein assay kit (Bio-Rad Laboratories, Hercules, CA). The lysates were centrifuged, and proteins $(25 \mu \mathrm{g})$ were subjected to electrophoresis on Tris-glycine SDS polyacrylamide gels. After blotting onto PVDF-membrane (Bio-Rad Laboratories), nonspecific binding sites were blocked using milk and incubations with primary antibodies were carried out overnight in Trisbuffered saline at $4^{\circ} \mathrm{C}$. Once washed, proteins were detected using enhanced chemiluminescence (Amersham Corporation, Arlington Heights, IL) according to the manufacturer's instructions. Equal extract loading was confirmed using actin expression, and expression levels were quantified using a densitometric analyzer.

Cell proliferation assays. To address potential dose-dependent effects on cytotoxicity, OSCC cells were treated with increasing concentrations of 17-AAG and analyzed using cell proliferation assays. After replicate plating a known number of single cells from each cell line into 12-well culture plates, the antiproliferative effect of 17-AAG and/or radiation on the growth profile of each OSCC cell line was examined. Twenty-four hours later, $100 \mathrm{nM}$ 17-AAG was added to one cell group. Control and 17-AAG-treated cells were then irradiated with a single dose of 4 Gy within 30 min of adding 17-AAG. Cells were harvested using trypsinization and counted with a hematocytometer (Kayagaki Irika Kogyo, Tokyo, Japan).

Clonogenic survival assay. Eight hundred cells were plated in triplicate in 35-mm tissue culture dishes. After $48 \mathrm{~h}, 17-\mathrm{AAG}$ $(100 \mathrm{nM})$ was added to the medium of one group of cells. Control and 17-AAG-treated cells were then irradiated with various doses $(2,4,6,8$, or $10 \mathrm{~Gy})$ using a linear accelerator (MBR.1520R, Hitachi Medico, Tokyo) at a source-to-target distance of $50 \mathrm{~cm}$, within $30 \mathrm{~min}$. Cell medium was changed to medium without $17-\mathrm{AAG} 24 \mathrm{~h}$ after radiation and incubated for $\sim 10$ days, allowing for colony formation. The colonies were fixed, stained with a solution of crystal violet and then enumerated. The survival fractions were calculated as a ratio of plating efficiencies for treated and untreated cells. These experiments were repeated at least three times. The linear quadratic model (17) was used to fit the cell survival data to the following equation: $\mathrm{Ln}$ [survival fraction $(\mathrm{SF})]=-\alpha \mathrm{D}-\beta \mathrm{D} 2$, where $D$ represents radiation dose (Gy) and $\alpha$ and $\beta$ correspond to the individual data. The parameters of $\alpha$ and $\beta$ were calculated for each curve. 

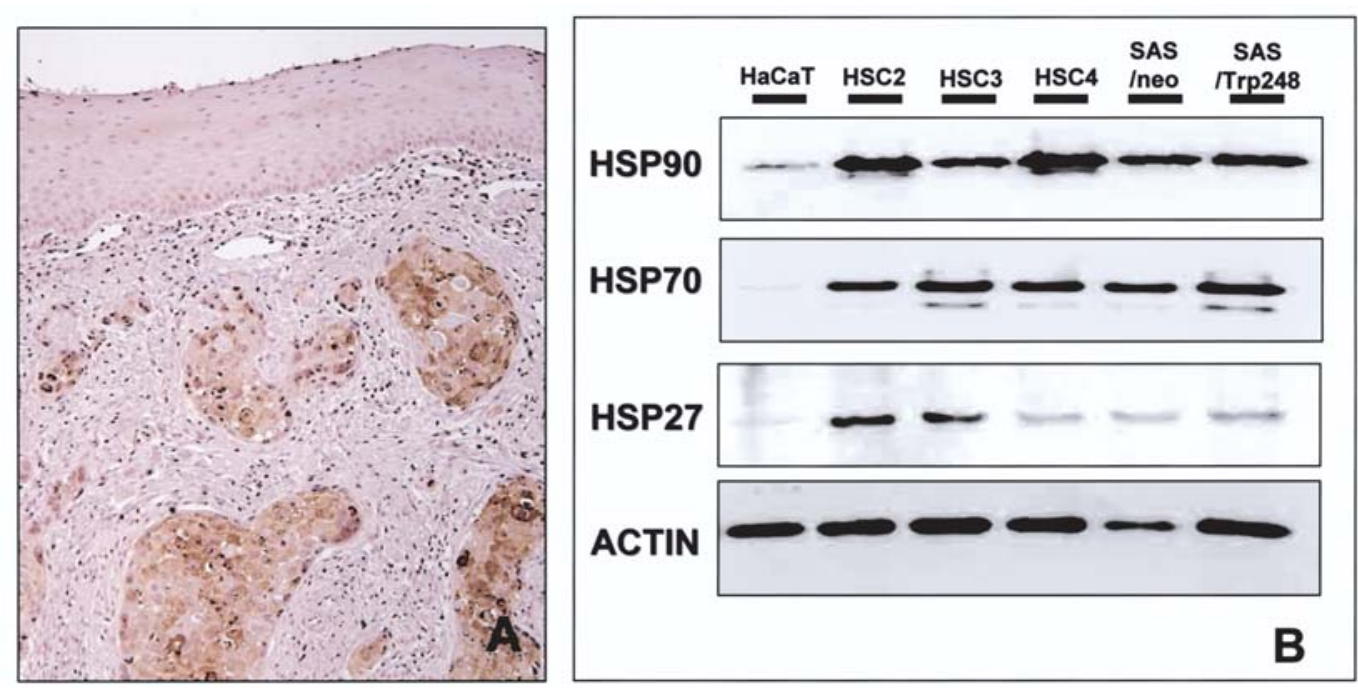

Figure 1. Expression of Hsp90 proteins in OSCC tissues and OSCC cell lines. A, immunohistochemical staining of Hsp90 in oral squamous cell carcinoma. Positive staining is seen in the cytoplasm of cancer cells (original magnification x100). B, Western blot analysis of Hsp27, Hsp70 and Hsp90 protein expression in normal keratinocyte cell line HaCaT and oral squamous cell carcinoma cell lines.

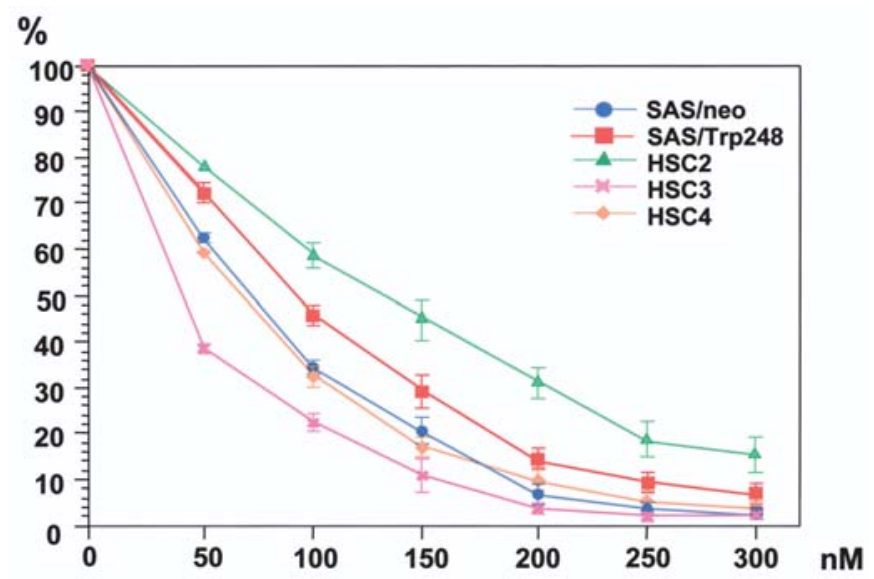

Figure 2. Dose-dependent growth inhibition effects of 17-allylamino-17demethoxygeldanamycin (17-AAG) in OSCC cell lines. Survival curves for OSCC cell lines after treatment with 17-AAG are based on the results of cell proliferation assays, after continuous drug exposure for $48 \mathrm{~h}$. Shown are the mean \pm SD values from a minimum of three independent experiments.

Detection of apoptosis. Assays for apoptosis detection and measurement were performed according to the manufacturer's instructions (Annexin V-FITC Apoptosis Detection kit; Oncogene Research, San Diego, CA). Briefly, phosphatidylserine is located on the cytoplasmic surface of the cell membrane in viable cells. However, apoptosis-induced alterations in phospholipid organization result in cell surface externalization of phosphatidylserine, where it can be detected with the anticoagulant Annexin V. Annexin V-FITC conjugate and propidium iodide were used in a flow cytometric method to distinguish between early apoptotic and necrotic or late apoptotic cells. SAS/neo and SAS/Trp248 cells were plated at a density of $5 \times 10^{5}$ cells/dish, incubated for $24 \mathrm{~h}$, treated with 17-AAG, and exposed to radiation. Cells were washed with PBS to remove the drug, fresh medium was added, and then allowed to recover in the incubator for designated time intervals after treatment. Spent media was collected on ice, in which attached cells were trypsinized and resuspended. Cells $(500,000)$ were transferred to a microcentrifuge tube, incubated with media-binding reagent and Annexin V-FITC, and centrifuged at $900 \mathrm{rpm}$ for $5 \mathrm{~min}$ at $4^{\circ} \mathrm{C}$. Cell media was removed and resuspended in binding buffer and propidium iodide. Samples were kept on ice in the dark until flow cytometry processing. Data from 10,000 events were collected on a Coulter Epics XL Flow Cytometer (Coulter Corp., Miami, FL) and analyzed using CellQuest/ModFit software.

Statistical analysis. All calculations were performed using the statistical computer program Statview 5.1 (Avacus, NC). The differences among treatment groups were examined by One-way ANOVA or Student's t-test. The differences in each cell cycle phase between each treatment were evaluated using the Mann-Whitney test. For each analysis, $\mathrm{p}<0.05$ was considered statistically significant.

\section{Results}

Expression of HSP90 proteins in OSCC tissues and OSCC cell lines. Normal epithelium was negative for Hsp90, while the cytoplasm of squamous cell carcinoma was positive and 18 out of 20 OSCC cases expressed Hsp90 (Fig. 1A). OSCC cell lines expressed Hsp27, Hsp70 and Hsp90 in high levels, and low protein expression was noted through Western blot analysis in the normal keratinocyte cell line, HaCaT (Fig. 1B).

17-AAG augments cell growth inhibition by radiation and enhances radiosensitivity in vitro. $17-\mathrm{AAG}$ is a dose-dependent inhibitor of cell growth in OSCC cell lines (HSC2, HSC3, HSC4, SAS/neo and SAS/Trp248), in vitro. Inhibition of cell growth occurs in response to increased dosages from 50 to $300 \mathrm{nM}$ 17-AAG for $24 \mathrm{~h}$ (Fig. 2). The effect of combined radiation and 17-AAG treatment was tested on OSCC cell line growth, where 17-AAG suppressed cell growth in a time-dependent fashion. 

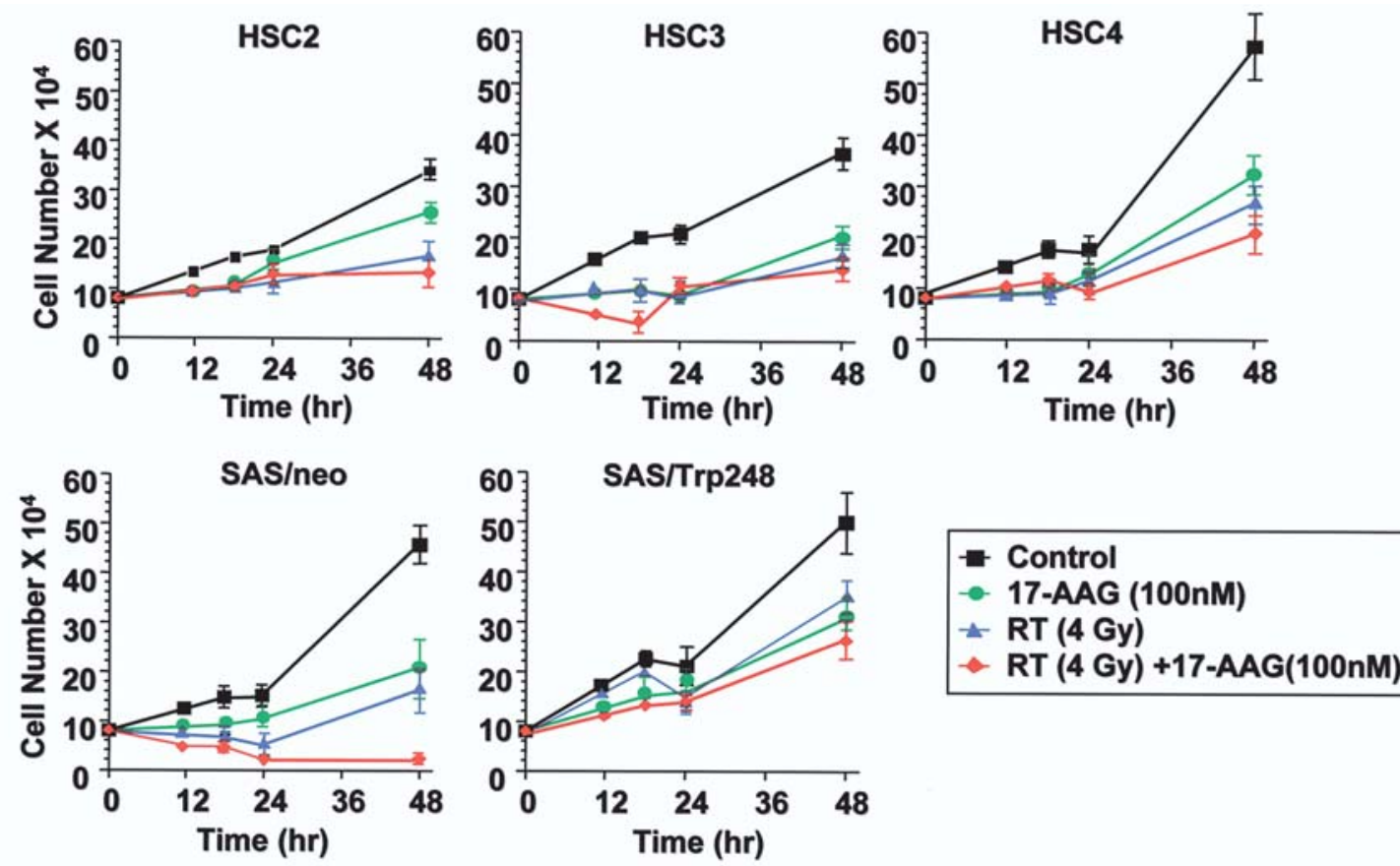

Figure 3. Additive growth inhibition by a combination of radiation and 17-AAG in the OSCC cell line. Known numbers of single cells were plated into culture dishes in Ham F12: Dulbecco's modified Eagle's medium containing 10\% FBS. After 24 h, 100 nM 17-AAG was added to the medium of 17-AAGtreated cells. Control and 17-AAG-treated cells were irradiated with a single dose of 4 Gy within 30 min of the addition of 17-AAG, as described in Materials and methods. Cancer cell lines were treated with 17-AAG $(100 \mathrm{nM})$, radiation (RT; 4 Gy), or a combination. Shown are the mean \pm SD values from three independent experiments.

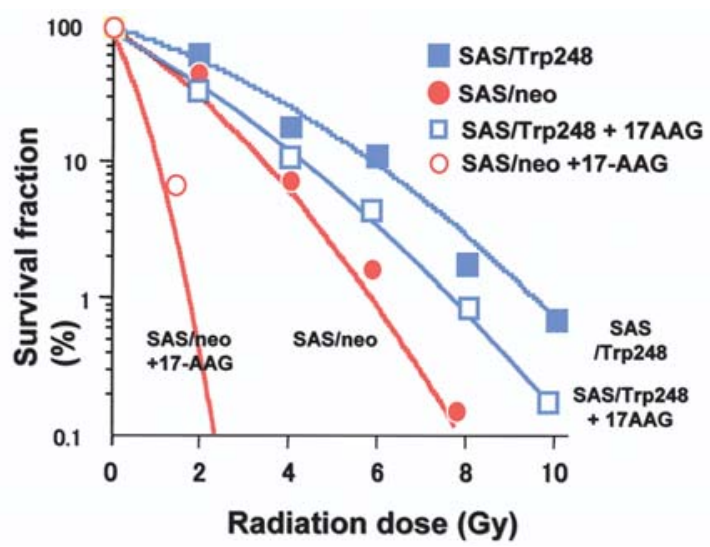

Figure 4. Clonogenic survival of two OSCC cell lines (SAS/neo and SAS/ Trp248) treated with radiation (RT) alone and in combination with 17-AAG $(100 \mathrm{nM})$. Cells $(800)$ were plated in triplicate in $35-\mathrm{mm}$ tissue culture dishes. After 48 h, $100 \mathrm{nM}$ 17-AAG was added to the medium of 17-AAGtreated cells. Control and 17-AAG-treated cells were irradiated with various single radiation doses $(2,4,6,8$ or $10 \mathrm{~Gy})$ as described in Materials and methods. Radiation sensitivity $\left(\mathrm{D}_{10}\right)$ improved by 1.5 - to 3.4 -fold with the addition of 17-AAG.

An inhibitory growth effect was observed in the SAS/neo cell line when 17-AAG (100 nM) and radiation (4 Gy) were combined. However, we did not find such an addictive effect with the combination of 17-AAG and radiation in HSC2, HSC3, HSC4 and SAS/Trp248 cell lines (Fig. 3). The effects of $24 \mathrm{~h}$ 17-AAG-pretreatment on the radiation response of SAS/neo and SAS/Trp248 cells were observed using a clonogenic cell survival assay. After 17-AAG-treatment (100 nM), cells were exposed to radiation doses as indicated $(2,4,6,8$

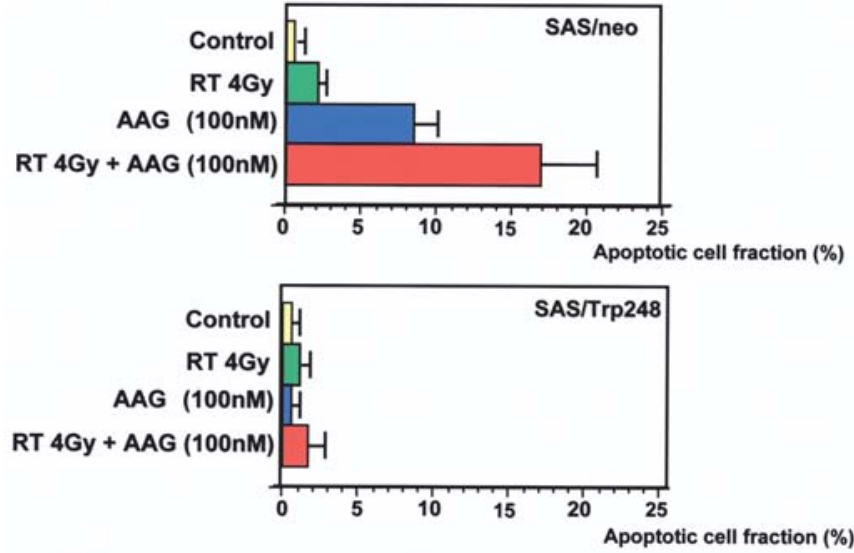

Figure. 5. Apoptosis induction with 17-AAG and/or radiation treatment RT. SAS/neo and SAS/Trp248 cells were treated with 17-AAG (100 nM) and exposed to radiation (4 Gy). Cells were harvested $24 \mathrm{~h}$ after exposure and Annexin V-FITC was added to the cell suspension followed by incubation at room temperature for $15 \mathrm{~min}$ in the dark. Samples were kept on ice in the dark and analyzed by flow cytometry using Cell Quest and ModFit software. Ten thousand events were collected for analysis.

or $10 \mathrm{~Gy}$ ). Colonies were stained and scored 10 days later, and the surviving fraction was plotted against the radiation dose (Gy). Curves were normalized to account for drug-induced cytotoxicity. The radiation dose required to cause a $10 \%\left(\mathrm{D}_{10}\right)$ survival fraction for each cell line decreased $(\mathrm{p}<0.05)$ in the presence of 17-AAG (SAS/neo, 3.4 to $1.0 \mathrm{~Gy}$; SAS/Trp248, 6.0 to $4.1 \mathrm{~Gy}$ ). Thus, the addition of 17-AAG enhanced the radiation sensitivity of both cell lines by 3.4 - to 1.5 -fold, respectively (Fig. 4, Table I). 


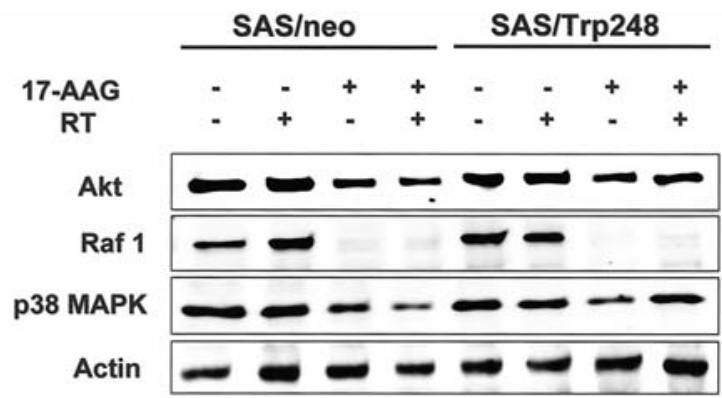

Figure 6. Effect of 17-AAG on the subcellular distribution of Akt, Raf-1 and p38 MAPK. Control or 17-AAG-treated (100 nM) OSCC cells (SAS/neo and SAS/Trp248) were irradiated (RT) with 4 Gy. After a 24-h incubation period, cells were lysed as described in Materials and methods. Lysates were then processed for immunoblotting using antibodies directed against DNA-PKcs, $\mathrm{Ku} 70$, and Ku86 as described above.

17-AAG induced programmed cell death in SAS/neo cell line. To address the mode of cell death induced by 17-AAG, SAS/neo and SAS/Trp248 cells were treated as described above and assayed for apoptosis. Cells were then treated with 17-AAG alone (100 nM) and/or radiation (4 Gy). Twenty-four hours after exposure, programmed cell death was measured using an Annexin V-FITC apoptosis detection protocol.

Exposure to $17-\mathrm{AAG}$ alone increased apoptosis to $\sim 8.3 \%$, while radiation exposure alone indicated about $2.1 \%$ apoptosis at $4 \mathrm{~Gy}$ of radiation in the SAS/neo cell line (Fig. 5). When cells were treated with $17-\mathrm{AAG}$ and radiation, SAS/neo cell line apoptosis was observed at $17.0 \%$. Apoptosis in the SAS/ Trp248 cell line, however, was not observed with 17-AAG alone or in combination with radiation, suggesting variable p53 status.

Effects of 17-AAG and/or radiation on multiple signaling pathways and cell cycle regulation. 17-AAG is directed toward a specific molecular target (Hsp90) and simultaneously inhibits multiple signaling pathways that cancer cells depend on for growth and survival. Therefore, we examined a series of signaling pathways using a combination of antibodies against such signaling factors. Through Western blot analysis we observed expression of proteins from pathways expected to be susceptible to 17-AAG. Although radiation and/or 17-AAG did not significantly affect p38/MAPK expression, $24 \mathrm{~h}$ 17-AAG (100 $\mathrm{nM})$ alone or in combination with radiation (4 Gy) induced significant suppression of Akt and Raf-1 in both SAS/neo and SAS/Trp248 cells (Fig. 6).

\section{Discussion}

New molecular target therapeutic agents inhibit specific intracellular factors in tumor cells and provide promising anticancer therapies. Recent efforts to develop agents that enhance tumor cell radiation sensitivity have focused on finding a specific molecule involved in regulating the cellular radiation response. There are numerous examples in which targeting a selected radiation response-associated molecule affects the radiation sensitivity of some tumor cell lines, but not others (25-27). Thus, it would seem that the effectiveness of target-based radiation sensitizers against solid neoplasms would be significantly constrained by intratumor heterogeneity.
Table I. Radiation survival parameters for SAS/neo and SAS/Trp248 combined with 17-AAG.

\begin{tabular}{lcc}
\hline Cell lines & $\mathrm{SF}_{2}$ & $\mathrm{D}_{10}$ \\
\hline SAS/neo & 0.45 & 3.4 \\
SAS/neo + 17AAG & 0.06 & 1.0 \\
SAS/Trp248 & 0.67 & 6.0 \\
SAS/Trp248 + 17AAG & 0.34 & 4.1 \\
\hline
\end{tabular}

$\mathrm{SF}_{2}$, survival fraction at $2 \mathrm{~Gy} . \mathrm{D}_{10}$, dose to give SF of $10 \%$.

In an attempt to increase the probability of enhanced tumor radiation sensitivity, we explored a multi-targeted approach using radiation sensitization, focusing on Hsp90 inhibition and its client proteins.

17-AAG represents agents that alter the intracellular function of multiple signaling factors shown to up-regulate prosurvival pathways $(9,28)$, including those that protect tumor cells from radiation cytotoxicity $(29,30)$. Additional evidence has shown that altering the functionality of intracellular Hsps, either via thermal stress or exposure to non-steroidal antiinflammatory agents, sensitizes tumor cells to therapeutic radiation cytotoxicity $(31,32)$. Through binding to the catalytic subdomain of Hsp90, 17-AAG also alters the chaperone functionality of key Hsps $(1,2,5)$. Therefore, it is plausible that 17-AAG, through abrogation of prosurvival pathways and alteration of Hsp90 conformation, may increase tumor cell death when combined with radiation.

In vitro tumor models using OSCC cell lines demonstrate that 17-AAG inhibited cell proliferation in a dose-dependent manner. 17-AAG (100 $\mathrm{nM})$ induced significant radiation sensitivity in SAS/neo cells that have wild-type p53 protein. In the other OSCC cell lines (HSC2, HSC3, HSC4 and SAS/Trp248), which have mutant-type p53 protein, 17-AAG inhibited cell growth; however, there was no significant enhancement of radiation sensitivity. Previous studies have shown that Hsp90 inhibitors, geldanamycin and 17-AAG, enhance in vitro the radiation sensitivity of cell lines from a variety of human tumors (33-35). This report evaluated human carcinoma cell lines that have wild-type p53 protein which enhance radiation sensitivity.

The mechanism of 17-AAG cytotoxicity and radiation sensitization is likely complex, involving multiple pathways including those related to programmed cell death and cell cycle regulation. Previous reports have described cellular p53 status in relation to radiation sensitivity $(22,36)$. The present study analyzed the effects of 17-AAG on the growth and clonogenic survival of human OSCC cell lines with differing p53 status. We have confirmed that 17-AAG could induce p53-dependent apoptosis in SAS/neo cells after radiation; however, these agents hinder apoptosis induction in SAS/ Trp248 cells. 17-AAG (100 nM) decreased the expected clonogenic survival of SAS/neo and SAS/Trp248 by $>3.4$ and 1.5-fold that after radiation, respectively. According to our experimental model, in contrast to independent p53 alterations, 17-AAG does not appear to be a radiation sensitizer of OSCC cells' mutant p53. Thus, our results concur with 
previous experiments that have shown that Hsp90 antagonist therapies reactivate mutant p53, making it incompatible (37). Others report that Hsp90 is required for both stabilization and reactivation of mutated $\mathrm{p} 53$, but it is not required for maintaining wild-type p53 (37).

Many cancer cells retain wild-type p53 and have persevered extensive DNA damage without undergoing apoptosis. On the other hand, cells whose p53 functions are disrupted lose their capacity to undergo p53-driven apoptosis (38). Based on the distinct p53 status of these SAS cell lines, we investigated to what extent apoptosis was involved in cell death after radiation, and if it was enhanced by 17-AAG. The OSCC cell lines with mutant p53 appeared to be resistant to radiation-induced apoptosis, and 17-AAG could not modify this apoptosis induction. In SAS/Trp248 cell lines that express mutated $\mathrm{p} 53$, apoptosis induction was totally abolished. Only the combination of 17-AAG and radiation triggered programmed cell death, inducing apoptosis in SAS/neo cell lines with wildtype p53. 17-AAG inhibits cell proliferation, consequently inducing radiation-treated SAS/ neo cells into G2/M. However, 17-AAG inhibits SAS/Trp248 cell proliferation and retards the release from $\mathrm{G} 0 / \mathrm{G} 1$ after radiation.

Based on our findings, Hsp90 therapy can be integrated with conventional therapies in patients with wild-type p53. The findings also demonstrate the importance of the precise characterization of the interaction between p53 mutants and stress proteins, which may shed valuable information on the fight against cancer via the p53 tumor suppressor pathway.

Although there are a considerable number of other Hsp90 client proteins, this study focused on the effects 17-AAG has on the levels of Akt, Raf-1, and P38 MAPK. These proteins are of particular interest because their inhibition has been associated with enhanced radiation sensitivity in some systems $(3,4,25,39,40)$. In both cell lines which have differing p53 status, Akt and Raf-1 were significantly reduced by $17-\mathrm{AAG}$. However, radiation and/or 17-AAG did not significantly affect the expression of p38/MAPK and Akt-1. To what extent a reduction in these proteins (Akt and Raf-1) contributes to enhanced radiation sensitivity is still unclear. There was no difference in protein reduction between SAS/neo and SAS/Trp248 cell lines, although there was a discrepancy in enhancement of radiation sensitivity. Therefore our findings, which concur with previous reports $(4,25,26)$, suggest that the radiation sensitivity effects of Raf-1 and Akt may depend on cell type.

\section{References}

1. Neckers L, Schulte TW and Mimnaugh E: Geldanamycin as a potential anti-cancer agent: its molecular target and biochemical activity. Invest New Drugs 17: 361-373, 1999.

2. Neckers L: HSP90 inhibitors as novel cancer chemotherapeutic agents. Trends Mol Med 8: 55-61, 2002.

3. Tanno S, Yanagawa N and Habiro A: Serine/threonine kinase AKT is frequently activated in human bile duct cancer and is associated with increased radioresistance. Cancer Res 64: 3486-3490, 2004.

4. Pirollo K, Hao Z, Rait A, Ho C and Chang E: Evidence supporting a signal transduction pathway leading to the radiation-resistant phenotype in human tumor cells. Biochem Biophys Res Commun 230: 196-201, 1997.

5. Ferrarini M, Heltai S, Zocchi MR and Rugarli C: Unusual expression and localization of heat-shock proteins in human tumor cells. Int J Cancer 51: 613-619, 1992.
6. Ito T, Kawabe R, Kurasono Y, Hara M, Kitamura H, Fujita K and Kanisawa M: Expression of heat shock proteins in squamous cell carcinoma of the tongue: an immunohistochemical study. $\mathbf{J}$ Oral Pathol Med 27: 18-22, 1998.

7. Hostein I, Robertson D, Di Stefano F, Workman P and Clarke PA: Inhibition of signal transduction by the Hsp90 inhibitor 17allylamino-17-demethoxygeldanamycin results in cytostasis and apoptosis. Cancer Res 61: 4003-4009, 2001.

8. Ramanathan RK, Trump DL, Eiseman JL, Belani CP, Agarwala SS, Zuhowski EG, Lan J, Potter DM, Ivy SP, Ramalingam S, Brufsky AM, Wong MK, Tutchko S and Egorin MJ: Phase I pharmacokinetic-pharmacodynamic study of 17-(allylamino)-17-demethoxygeldanamycin (17AAG, NSC 330507 ), a novel inhibitor of heat shock protein 90, in patients with refractory advanced cancers. Clin Cancer Res 11: 3385-3391, 2005.

9. Prodromou C, Roe SM, O'Brien R, Ladbury JE, Piper PW and Pearl LH: Identification and structural characterization of the ATP/ADP-binding site in the Hsp90 molecular chaperone. Cell 90: $65-75,1997$.

10. Obermann WM, Sondermann H, Russo AA, Pavletich NP and Hartl FU: In vivo function of HSP90 is dependent on ATP binding and ATP hydrolysis. J Cell Biol 143: 901-910, 1998.

11. Workman P and Maloney A: HSP90 as a new therapeutic target for cancer therapy: the story unfolds. Expert Opin Biol Ther 2: 3-24, 2002.

12. Chiosis G, Huezo H, Rosen N, Mimnaugh E, Whitesell L and Neckers L: 17AAG: low target binding affinity and potent cell activity: finding an explanation. Mol Cancer Ther 2: 123-129, 2003.

13. Solit DB, Basso AD, Olshen AB, Scher HI and Rosen N: Inhibition of heat shock protein 90 function down-regulates Akt1 kinase and sensitizes tumors to Taxol. Cancer Res 63: 2139-2144, 2003.

14. Doong H, Rizzom K, Fangm S, Kulpam V, Weissmanm AM and Kohn EC: CAIR-1/BAG-3 abrogates heat shock protein-70 chaperone complex-mediated protein degradation: accumulation of poly-ubiquitinated Hsp90 client proteins. J Biol Chem 278: 28490-28500, 2003

15. Workman P: Combinatorial attack on multistep oncogenesis by inhibiting the Hsp90 molecular chaperone. Cancer Lett 206: 149-157, 2004.

16. Calabrese C, Frank A, Maclean K and Gilbertson R: Medulloblastoma sensitivity to 17-allylamino-17-demethoxygeldanamycin requires MEK/ERKM. J Biol Chem 278: 24951-24959, 2003.

17. Russell J, Burgan W, Oswald K, Camphausen K and Tofilon P: Enhanced cell killing induced by the combination of radiation and the heat shock protein 90 inhibitor 17-allylamino-17demethoxygeldanamycin: a multitarget approach to radiosensitization. Clin Cancer Res 9: 3749-3755, 2003.

18. Harashima K, Akimoto T, Nonaka T, Tsuzuki K, Mitsuhashi N and Nakano T: Heat shock protein 90 (Hsp90) chaperone complex inhibitor, radicicol, potentiated radiation-induced cell killing in a hormone-sensitive prostate cancer cell line through degradation of the androgen receptor. Int J Radiat Biol 81: 63-76, 2005

19. Kobayashi S, Nantz R, Kitamura T, Higashikubo R and Horikoshi N: Combined inhibition of extracellular signal-regulated kinases and HSP90 sensitizes human colon carcinoma cells to ionizing radiation. Oncogene 24: 3011-3019, 2005.

20. Slichenmyer W, Nelson W, Slebos R and Kastan M: Loss of a p53-associated G1 checkpoint does not decrease cell survival following DNA damage. Cancer Res 53: 4164-4168, 1993.

21. Bristow R, Benchimol S and Hill R: The p53 gene as a modifier of intrinsic radiosensitivity: implications for radiotherapy. Radiother Oncol 40: 197-223, 1996.

22. Bohnke A, Westphal F, Schmidt A, El-Awady RA and Dahm-Daphi J: Role of p53 mutations, protein function and DNA damage for the radiosensitivity of human tumor cells. Int $\mathbf{J}$ Radiat Biol 80: 53-63, 2004.

23. Fan S, El-Deiry WS, Bae I, Freeman J, Jondle D, Bhatia K, Fornace AJ Jr, Magrath I, Kohn KW and O'Connor PM: p53 gene mutations are associated with decreased sensitivity of human lymphoma cells to DNA damaging agents. Cancer Res 54: 5824-5830, 1994.

24. Ota I, Ohnishi K, Takahashi A, Yane K, Kanata H, Miyahara H, Ohnishi T and Hosoi H: Transfection with mutant p53 gene inhibits heat-induced apoptosis in a head and neck cell line of human squamous cell carcinoma. Int J Radiat Oncol Biol Phys 47: 495-501, 2000. 
25. Gupta A, Bakanauskas V, Cerniglia G, et al: The Ras radiation resistance pathway. Cancer Res 61: 4278-4282, 2001.

26. Tezuka M, Watanabe $\mathrm{H}$ and Nakamura S: Antiapoptotic activity is dispensable for insulin-like growth factor I receptor-mediated clonogenic radioresistance after gamma-irradiation. Clin Cancer Res 7: 3206-3214, 2001.

27. Li J, Wang Y, Sun Y and Lawrence T: Wild-type TP53 inhibits G(2)-phase checkpoint abrogation and radiosensitization induced by PD0166285, a WEE1 kinase inhibitor. Radiat Res 157: 322-330, 2002.

28. Stebbins CE, Russo AA, Schneider C, Rosen N, Hartl FU and Pavletich NP: Crystal structure of an Hsp90-geldanamycin complex: targeting of a protein chaperone by an antitumor agent. Cell 89: 239-250, 1997.

29. Sartor CI: Epidermal growth factor family receptors and inhibitors: radiation response modulators. Semin Radiat Oncol 13: 22-30, 2003

30. Isaacs JS, Jung YJ, Mimnaugh EG, Martinez A, Cuttitta F and Neckers LM: Hsp90 regulates a von Hippel Lindau-independent hypoxia-inducible factor-1-degradative pathway. J Biol Chem 277: 29936-29944, 2002.

31. Bradbury CM, Markovina S, Wei J, Renee LM, Zoberi I, Horikoshi N and Gius D: The role of NF-B as one downstream target of p38 MAPK in the process of indomethacin-induced radiosensitization. Cancer Res 61: 7689-7696, 2001.

32. Palayoor ST, Bump EA, Calderwood SK, Bartol S and Coleman CN: Combined antitumor effect of radiation and ibuprofen in human prostate carcinoma cells. Clin Cancer Res 4: 763-771, 1998.

33. Enmon R, Yang W and Ballangrud A: Combination treatment with 17-allylamino-17-demethoxygeldanamycin and acute irradiation produces supra-additive growth suppression in human prostate carcinoma spheroids. Cancer Res 63: 8393-8399, 2003.
34. Machida H, Matsumoto Y, Shirai M and Kubota N: Geldanamycin, an inhibitor of Hsp90, sensitizes human tumor cells to radiation. Int J Radiat Biol 79: 973-980, 2003.

35. Bisht K, Bradbury M and Mattson D: Geldanamycin and 17allylamino-17-demethoxygeldanamycin potentiate the in vitro and in vivo radiation response of cervical tumor cells via the heat shock protein 90-mediated intracellular signaling and cytotoxicity. Cancer Res 63: 8984-8995, 2003.

36. Scott SL, Earle JD and Gumerlock PH: Functional p53 increases prostate cancer cell survival after exposure to fractionated doses of ionizing radiation. Cancer Res 63: 7190-7196, 2003.

37. Muller P, Ceskova P and Vojtesek B: Hsp90 is essential for restoring cellular functions of temperature-sensitive p 53 mutant protein but not for stabilization and activation of wild-type p53: implications for cancer therapy. J Biol Chem 280: 6682-6691, 2005.

38. Asker C, Wiman K and Selivanova G: p53-induced apoptosis as a safeguard against cancer. Biochem Biophys Res Commun 265: 1-6, 1999.

39. Dent P, Yacoub A, Contessa J, Caron R, Amorino G, Valerie K, Hagan MP, Grant S and Schmidt-Ullrich R: Stress and radiationinduced activation of multiple intracellular signaling pathways. Radiat Res 159: 283-300, 2003.

40. Kumar P, Miller AI and Polverini PJ: p38 MAPK mediates gamma-irradiation-induced endothelial cell apoptosis, and vascular endothelial growth factor protects endothelial cells through the phosphoinositide 3-kinase-Akt-Bcl-2 pathway. J Biol Chem 279: 43352-43360, 2004. 